\title{
Caracterização física e físico-química de frutos de mangabeira (Hancornia speciosa Gomes) no oeste da Bahia
}

\author{
${ }^{1}$ UFV, Rio Paranaíba, MG. E-mail: roxana.mendes@hotmail.com (Autor correspondente) \\ ${ }^{2}$ IFTO, Dianópolis, TO. E-mail: j.avelino86@hotmail.com \\ ${ }^{3}$ UNEB, Barreiras, BA. E-mail: fabiococozza@uneb.br
}

Roxana S. M. Nascimento ${ }^{1}$, José A. Cardoso² \& Fabio D. M. Cocozza

\section{Palavras-chave:}

fruteira nativa

cerrado

caracterização físico-química

\begin{abstract}
R E S U M O
Comparada a outras frutas nativas do Nordeste, a mangaba é utilizada pela agricultura destacando-se no bioma Cerrado pelo aproveitamento alimentar. Com este trabalho objetivou-se avaliar a caracterização física e físicoquímica de frutos de mangabeira da região Oeste da Bahia. Foram amostrados 300 frutos de dez matrizes, coletados em uma área dos municípios de Angical, Barreiras e São Desidério e conduzidos ao Laboratório de Sementes da UNEB. Foram considerados, para as avaliações físicas, os diâmetros longitudinal e transversal do fruto; o peso do fruto; peso total de semente por fruto; peso de polpa e casca; rendimento de polpa e casca; já para as avaliações físico-químicas a polpa dos frutos foi submetida às determinações do pH; do teor de sólidos solúveis (SS); acidez titulável (AT) e o ratio. Para as variáveis diâmetro longitudinal e transversal, peso de polpa e casca, peso de sementes, peso de fruto e rendimento de polpa e casca as médias foram, respectivamente, 32,34 e $31,87 \mathrm{~mm}, 14,77,2,40$ e $17,17 \mathrm{~g}$ e $85,93 \%$ porém no que se refere ao $\mathrm{pH}$, SS, AT e ratio (SS/AT), as médias foram $3,93,17,04^{\circ}$ Brix, 0,98 e 18,62 cujos resultados indicaram que há diferença estatística significativa entre todas as variáveis avaliadas.
\end{abstract}

Key words: native fruit tree cerrado physicochemical characterization

\section{Physical and physicochemical characterization of 'mangabeira' fruits (Hancornia speciosa Gomes) in Western Bahia}

\begin{abstract}
A B S T R A C T
The 'mangaba' is widely used by agribusiness, as compared to other native fruits of Northeast, especially in the Cerrado biome for food utilization. This study aimed to evaluate the physical and physicochemical characterization of 'mangabeira' fruits in the western region of Bahia. Three hundred fruits were sampled from ten matrices, which were collected in an area of the municipalities of Angical, Barreiras and St Desiderio and conducted to the Seed Laboratory of UNEB. For the physical assessments, longitudinal and transversal diameters of the fruit, fruit weight, total seed weight per fruit, weight of the pulp and peel, and yield of pulp and peel were considered. As for the physicochemical evaluations, the fruit pulp was subjected to the determinations of $\mathrm{pH}$, soluble solids content, titratable acidity and ratio. For the variables, longitudinal and transversal diameters, the weight of pulp and peel, seed weight, fruit weight and yield of pulp and peel, the means were, respectively, 32.34 and $31.87 \mathrm{~mm}, 14.77,2.40$ and $17.17 \mathrm{~g}$ and $85.93 \%$. With regard to $\mathrm{pH}$, SS, AT and ratio (SS/AT), the means were $3.93,17.04^{\circ} \mathrm{Brix}, 0.98$ and 18.62. The results indicated that there are statistically significant differences between all variables evaluated.
\end{abstract}

\section{INTRODUÇÃo}

A mangabeira (Hancornia speciosa Gomes) é uma fruteira pertencente à família Apocynaceae, nativa do Brasil e está presente nas regiões Centro-Oeste, Sudeste, Norte e Nordeste nas áreas do cerrado e caatinga. A frutificação ocorre entre outubro e dezembro, porém a quase totalidade da produção nacional deste fruto se encontra nas áreas de tabuleiros e baixadas litorâneas do Nordeste (Venturini Filho, 2010).

Os frutos são do tipo baga, de tamanho, forma e cores variados, geralmente elipsoidais ou arredondados, variando de 2,5 a 6,0 cm de diâmetro, exocarpo amarelado ou esverdeado com pigmentação vermelha ou sem pigmentação, polpa amarela, bastante suave, adocicada, carnoso-viscosa, ácida (Ganga, et al., 2009; Venturini Filho, 2010), contendo, em geral, de 2 a 15 ou até 30 sementes discoides, achatadas e com coloração castanho-clara, de 7 a $8 \mathrm{~mm}$ de diâmetro (Lederman et al., 2000). Apresentam boa digestibilidade e valor nutritivo, com teor de proteína superior ao da maioria das frutas utilizadas comercialmente (Vieira Neto, 2001).

Existe um enorme potencial para exploração de frutas nativas no setor agroindustrial brasileiro (Hansen et al., 2013). Em função do excelente aroma e sabor dos frutos, a mangaba é muito utilizada pela agroindústria quando comparada a outras frutas nativas do Nordeste (Lederman et al., 2000). Assim, seus frutos podem ser consumidos in natura ou processados na 
forma de sorvete, suco, geleia, doces e licor (Borges et al., 2000). Apesar do grande potencial da mangabeira para a exploração agroindustrial (Ávila et al., 2010) a produção não tem atendido à demanda uma vez que esta espécie é explorada de forma extrativista, responsável que é pela maior parte da produção desses frutos (Vieira Neto, 2001). Desta forma, a mangaba, apesar do grande potencial econômico, é consumida apenas em sua área de ocorrência (Lederman et al., 2000).

As características físicas e físico-químicas dos frutos sofrem influência das condições edafoclimáticas, dos tratos culturais, época de colheita, constituição genética, estádio de maturação e do tratamento pós-colheita, entre outros (Fagundes \& Yamanishi, 2001). Tais características são fatores de qualidade de fundamental importância à utilização e comercialização da polpa dos frutos e para elaboração de produtos industrializados (Chitarra \& Chitarra, 2005). Por outro lado, a caracterização física dos frutos tem grande importância quando se refere à determinação da variabilidade genética de uma espécie que pode subsidiar programas de melhoramento genético, bem como sua relação com os fatores ambientais (Carvalho et al., 2003).

Assim, objetivou-se, com este trabalho, determinar as características físicas e físico-químicas de frutos de mangabeira in natura, no estágio de maturação maduro.

\section{Material e Métodos}

As coletas dos frutos foram realizadas em três áreas situadas nos municípios de São Desidério, Barreiras e Angical, no estado da Bahia, região Oeste, em outubro de 2009. O clima da região se caracteriza, conforme Thornthwaite \& Mather, como subúmido seco - C1d'A' - apresentando verão chuvoso e inverno extremamente seco, com uma precipitação pluvial anual de 700 a $2000 \mathrm{~mm}$ (AIBA, 2004) concentrada entre os meses de outubro e abril apresentando, entretanto, alta probabilidade de veranicos durante a estação chuvosa. As condições climáticas desta região variam bastante porém a região Oeste apresenta clima severo, baixa precipitação anual distribuída em curto período do ano e verões quentes e chuvosos. As temperaturas médias mínima e máxima anuais são, respectivamente, 14 e $34^{\circ} \mathrm{C}$. A umidade relativa do ar normal em Barreiras varia de $47,1 \%$ em setembro a $80,3 \%$ no mês de março alcançando uma média anual normal equivalente a $68 \%$. A região apresenta luminosidade equivalente a $3000 \mathrm{~h} \mathrm{ano}^{-1}$ (AIBA, 2004) além de vegetação de Cerrado e solo arenoso.

Os frutos foram coletados de exemplares com altura média de $5 \mathrm{~m}$ e escolhidas dez matrizes (Tabela 1 ) de onde foram retirados, no estádio de maturação maduro, 30 frutos por matriz os quais foram, posteriormente, acondicionados em sacos de polietileno e levados ao Laboratório de Sementes da Universidade do Estado da Bahia - UNEB/Barreiras - BA para realização das análises. De acordo com Cruz \& Carvalho (2002) são necessários 100 frutos, no mínimo, para proceder às avaliações biométricas; portanto, foram selecionados, aleatoriamente, 300 frutos maduros e morfologicamente perfeitos.
Tabela 1. Coordenadas e locais de coleta das matrizes de mangabeira

\begin{tabular}{clcc}
\hline Planta & \multicolumn{1}{c}{ Local } & \multicolumn{1}{c}{ Coordenada } & Altitude (m) \\
1 & Barreiras & $12^{\circ} 10^{\prime} 59,8^{\prime \prime} / 45^{\circ} 10^{\prime} 39,0^{\prime \prime}$ & 532 \\
2 & Barreiras & $12^{\circ} 12^{\prime} 00,5^{\prime \prime} / 45^{\circ} 11^{\prime} 54,1^{\prime \prime}$ & 550 \\
3 & Barreiras & $12^{\circ} 12^{\prime} 11,7^{\prime \prime} / 45^{\circ} 12^{\prime} 24,2^{\prime \prime}$ & 544 \\
4 & Angical & $11^{\circ} 45^{\prime} 80,9^{\prime \prime} / 44^{\circ} 37^{\prime} 23,0^{\prime \prime}$ & 447 \\
5 & Angical & $11^{\circ} 45^{\prime} 82,2^{\prime \prime} / 44^{\circ} 37^{\prime} 21,9^{\prime \prime}$ & 433 \\
6 & Angical & $11^{\circ} 45^{\prime} 82,9^{\prime \prime} / 44^{\circ} 37^{\prime} 20,5^{\prime \prime}$ & 420 \\
7 & Angical & $11^{\circ} 45^{\prime} 86,4^{\prime \prime} / 44^{\circ} 37^{\prime} 22,4^{\prime \prime}$ & 450 \\
8 & São Desidério & $12^{\circ} 41^{\prime} 18,6^{\prime \prime} / 44^{\circ} 59^{\prime} 54,8^{\prime \prime}$ & 693 \\
9 & São Desidério & $12^{\circ} 41^{\prime} 18,6^{\prime \prime} / 44^{\circ} 59^{\prime} 54,8^{\prime \prime}$ & 693 \\
10 & São Desidério & $12^{\circ} 43^{\prime} 28,3^{\prime \prime} / 44^{\circ} 57^{\prime} 05,0^{\prime \prime}$ & 500 \\
\hline
\end{tabular}

As análises físicas foram desenvolvidas individualizadas por fruto, conforme a metodologia da (AOAC, 1990). Em seguida, foram determinados o peso dos frutos em grama, utilizando-se balança de precisão digital; diâmetros longitudinal e transversal do fruto, medidos com paquímetro digital; número de sementes por fruto; peso médio de sementes por fruto; peso da polpa e da casca e rendimento de polpa, em porcentagem, obtido pela divisão entre o peso da polpa e o peso do fruto multiplicado por 100.

Para as avaliações físico-químicas os frutos de mangabeira foram coletados e sua polpa avaliada no estádio de maturação maduro. Foram coletados, aleatoriamente, 30 frutos dos quatro quadrantes de uma única matriz, imediatamente após se desprenderem da planta; em seguida, os mesmos foram transportados para o laboratório, acondicionados em caixas de isopor e posteriormente separados em lotes. Para obtenção da polpa os frutos selecionados foram macerados em uma peneira e a polpa envasada em sacos de polietileno, identificada e mantida em refrigeração $\left(-18^{\circ} \mathrm{C}\right) \mathrm{em}$ freezer durante as determinações físico-químicas. A polpa de cada matriz foi dividida em dez lotes iguais e cada lote foi composto pela polpa de três frutos. Os lotes representaram as repetições e as matrizes foram consideradas tratamentos.

Após o descongelamento da polpa dos frutos da mangabeira foram realizadas as avaliações físico-químicas. Os sólidos solúveis foram determinados através de leitura refratométrica direta, em refratômetro digital, expressa diretamente em ${ }^{\circ}$ Brix. O potencial hidrogeniônico $(\mathrm{pH})$ foi determinado pelo método potenciométrico em peagâmetro com auxílio de um medidor digital calibrando-se o potenciômetro através das soluções tampão (pH 4,0 e 7,0) (AOAC, 1990). A acidez titulável foi obtida por titulometria com solução padronizada de hidróxido de sódio a $0,1 \mathrm{~N}$ tendo, como indicador, o ponto de viragem da fenolftaleína. Os valores foram expressos em porcentagem, conforme metodologia do IAL (1985). Foi determinada a razão sólidos solúveis/acidez titulável.

Utilizou-se um delineamento experimental inteiramente casualizado em que os dados obtidos nas análises físicas e físicoquímicas foram submetidos à análise de variância pelo teste $\mathrm{F}$ enquanto as médias foram comparadas pelo teste de Tukey a 0,05 de probabilidade.

\section{Resultados e Discussão}

O diâmetro longitudinal e o transversal dos frutos de mangabeira diferiram em alguns níveis avaliados apresentando, 
no entanto como valor máximo do diâmetro longitudinal, 38,44 mm e o valor mínimo $24,89 \mathrm{~mm}$, com relação ao diâmetro transversal, tais características apresentaram os seguintes valores, respectivamente, 35,74 e 25,20 mm (Tabela 2). Esses resultados são equivalentes aos encontrados por Ganga et al. (2010) em estudo com mangabeiras de populações naturais do Cerrado, e relataram os valores de 37,30 mm para o diâmetro longitudinal e diâmetro transversal de $34,0 \mathrm{~mm}$; já Souza et al. (2007) observaram, estudando frutos de diferentes clones de mangabeiras, valores médios de 38,27 e 34,66 mm para os diâmetros longitudinal e transversal, respectivamente.

Para o mercado de frutas frescas, os frutos mais pesados e, consequentemente, os de maior tamanho, são mais atrativos aos consumidores, conforme Chitarra \& Chitarra (2005). Desta forma, o peso dos frutos foi diferente na maioria dos níveis avaliados, com média geral de 17,17 g, de acordo com a Tabela 2; apesar disto, 21,25 g corresponderam ao peso do fruto de maior massa e 8,56 g, ao fruto de menor massa (Tabela 2). Considerando essa característica merecem destaque as matrizes $4,8,9$ e 10 por apresentarem os frutos mais pesados. Ganga et al. (2010) constataram que o peso médio dos frutos foi de 27,88 g, apresentando, entretanto, como valor mínimo, 2,76 g e valor máximo 154,05 g, ainda Souza et al. (2007) encontraram o valor médio para peso do fruto de $25,74 \mathrm{~g}$. Segundo Vieira \& Gusmão (2008) e Santos et al. (2009) as variações no peso dos frutos podem ser decorrentes de variabilidade genética ou de variações ambientais devido às diferentes localidades geográficas.

Conforme a Tabela 2 ficou constatado que para a variável polpa e casca dos frutos houve diferença considerável com valores variando entre 7,06 a 22,03 g, apresentando média geral de $14,77 \mathrm{~g}$. Ganga et al. (2010) encontraram o valor de 27,88 g para esta característica. O peso de polpa e casca é o atributo físico de maior importância para a exploração econômica, principalmente no que se refere ao processamento de frutos. Merecem destaque as matrizes 8, 9 e 10.

Nos frutos avaliados o rendimento de polpa e casca variou de 81,47 a $93,05 \%$ da massa do fruto, com média de $85,93 \%$ (Tabela 2). Porém a percentagem média de polpa e casca em frutos de mangabeira encontrada por Ganga et al. (2010) foi de $82,68 \%$. O rendimento da polpa é um parâmetro de qualidade importantíssimo para a indústria de concentrados, purês, doces em massa, néctares etc. (Chitarra \& Chitarra, 2005). Segundo Lima et al. (2002) os frutos que apresentam rendimento em polpa superior a $50 \%$ demonstram condições adequadas para comercialização. As pesquisas de 1985 a 2003 têm destacado a mangaba como fruto que apresenta excelente potencial para industrialização de vez que possui, além de seu sabor exótico, rendimento em polpa superior a $70 \%$.

O número de sementes por fruto foi bastante variável apresentando uma variação de 1 a 29 sementes/fruto (Tabela 2). Souza et al. (2007) obtiveram o número médio de sementes de 14,80 e Ganga et al. (2010) obtiveram valor médio de 13,40 sementes. A média do peso de sementes por fruto foi de 2,40 g, de acordo com a Tabela 2, já Ganga et al. (2010) constataram o valor de peso médio de sementes de $3,88 \mathrm{~g}$. O número e a percentagem de sementes estão relacionados com o tamanho do fruto, consequentemente com o rendimento e também com a qualidade do produto (Chitarra \& Chitarra, 2005).

Observou-se, na Tabela 3, correlação positiva para todas as variáveis físicas dos frutos de mangabeira, sendo todas significativas estatisticamente com exceção das variáveis peso fruto $\mathrm{x}$ número de sementes/frutos, polpa e casca $\mathrm{x}$ número de sementes/frutos e número de sementes/frutos $\mathrm{x}$ diâmetro

Tabela 3. Correlação para as variáveis físicas dos frutos das matrizes de mangabeira

\begin{tabular}{|c|c|c|}
\hline Correlação & $\begin{array}{l}\text { Coef. } \\
\text { Corr (r) }\end{array}$ & Signif. \\
\hline Peso de fruto $x$ média de sementes por fruto & 0,749 & \\
\hline Peso de fruto x polpa e Casca & 0,988 & ** \\
\hline Peso de fruto $\mathrm{x}$ número de sementes por frutos & 0,571 & ns \\
\hline Peso de fruto $\mathrm{x}$ diâmetro longitudinal & 0,959 & ** \\
\hline Peso de fruto $x$ diâmetro transversal & 0,963 & ** \\
\hline Média de sementes por fruto $\mathrm{x}$ polpa e casca & 0,640 & * \\
\hline Média de sementes por fruto $\mathrm{x}$ número de sementes por frutos & 0,910 & ** \\
\hline Média de sementes por fruto $\mathrm{x}$ diâmetro longitudinal & 0,791 & ** \\
\hline Média de sementes por fruto $\mathrm{x}$ diâmetrotransversal & 0,690 & * \\
\hline Polpa e casca x número de sementes por frutos & 0,454 & ns \\
\hline Polpa e casca $x$ diâmetro longitudinal & 0,932 & ** \\
\hline Polpa e casca x diâmetro transversal & 0,959 & ** \\
\hline Número de sementes por frutos $\mathrm{x}$ diâmetro longitudinal & 0,686 & * \\
\hline Número de sementes por frutos $\mathrm{x}$ diâmetro transversal & 0,553 & ns \\
\hline Diâmetro longitudinal $x$ diâmetro transversal & 0,955 & ** \\
\hline
\end{tabular}

** Significativo ao nível de 0,01 de probabilidade $(p<0,01),{ }^{*}$ Significativo ao nível de 0,05 de probabilidade $(0.01 \leq p<0.05)$, ns - Não significativo

Tabela 2. Características físicas dos frutos das matrizes de Hancornia speciosa (mangabeira)

\begin{tabular}{|c|c|c|c|c|c|c|c|}
\hline \multicolumn{2}{|c|}{ Número } & \multicolumn{2}{|c|}{ Diâmetro (mm) } & \multicolumn{3}{|c|}{ Peso (g) } & \multirow{2}{*}{$\begin{array}{l}\text { Rendimento \% } \\
\text { (Polpa e casca) }\end{array}$} \\
\hline Matriz & Frutos & Long. & Trans. & Polpa e casca & Sementes & Frutos & \\
\hline 2 & 30 & $33,80 \mathrm{~b}$ & 32,12 bc & 15,24 bc & $1,20 \mathrm{e}$ & $16,44 \mathrm{c}$ & $93,05 \mathrm{a}$ \\
\hline 4 & 30 & $33,59 \mathrm{~b}$ & $35,05 \mathrm{a}$ & $18,19 b$ & $2,40 \mathrm{~cd}$ & $20,59 \mathrm{~b}$ & $88,41 b c$ \\
\hline 5 & 30 & $24,89 d$ & $25,20 \mathrm{~d}$ & $7,06 \mathrm{~d}$ & $1,50 \mathrm{de}$ & $8,56 d$ & 82,34 de \\
\hline 6 & 30 & $34,19 \mathrm{~b}$ & $33,85 a b$ & 15,07 bc & $2,60 \mathrm{bc}$ & 17,67 bc & 84,51 cde \\
\hline 9 & 30 & $37,91 \mathrm{a}$ & $34,19 a b$ & $17,31 b$ & $3,74 \mathrm{a}$ & $21,05 \mathrm{~b}$ & $82,48 \mathrm{de}$ \\
\hline \multirow[t]{3}{*}{10} & 30 & $37,28 \mathrm{a}$ & $34,64 \mathrm{a}$ & $17,13 b$ & $4,12 \mathrm{a}$ & $21,25 b$ & $81,47 \mathrm{e}$ \\
\hline & $M G$ & 32,34 & 31,87 & 14,77 & 2,40 & 17,17 & 85,93 \\
\hline & CV\% & 10,34 & 9,46 & 27,33 & 22,26 & 26,34 & 5,56 \\
\hline
\end{tabular}

Médias seguidas de letras diferentes nas colunas diferem pelo teste de Tukey, a nível de 0,05 de significância 
transversal. Correlações positivas indicam que as duas características são beneficiadas ou prejudicadas pelas mesmas causas de variação.

Os maiores valores de correlações observados foram para o peso fruto x polpa e casca $(0,988)$, peso fruto $\mathrm{x}$ diâmetro longitudinal $(0,959)$, peso fruto $\mathrm{x}$ diâmetro transversal $(0,963)$, média de sementes/fruto $\mathrm{x}$ número de sementes/ frutos $(0,910)$, polpa e casca x diâmetro longitudinal $(0,932)$, polpa e casca x diâmetro transversal $(0,959)$, diâmetro longitudinal x diâmetro transversal $(0,955)$, indicando que, quanto maiores o tamanho do fruto, o diâmetro transversal e longitudinal maiores também o rendimento de polpa e a casca; portanto, o valor de uma variável é diretamente proporcional ao valor da outra.

Os frutos de mangabeira apresentaram potencial hidrogeniônico variando entre 3,45 e 4,39, com valor médio de 3,93 (Tabela 4) concordando, então, com o valor encontrado por Moura et al. (2002) que revelou um pH de 3,30 em frutos do Rio Grande do Norte, e assemelhando-se ao citado por Carnelossi et al. (2004) estudando a caracterização pós-colheita de frutos de mangabeira de caída e de vez da região de Itaporanga D Ajuda - SE, constatando um $\mathrm{pH}$ de 3,50, e ao citado por Santos et al. (2012) que analisaram frutos físico-quimicamente, cujo valor encontrado foi 3,07. Assim, de acordo com Azeredo \& Brito (2004) os frutos com pH abaixo de 4,50 são classificados como muito ácidos.

Nos valores de SS houve uma variação de 15,34 a 18,70 ºrix, média geral de $17,04^{\circ}$ Brix, conforme a Tabela 4 . Os resultados encontrados foram superiores aos reportados por Santos et al. (2012) de $14,83^{\circ}$ Brix e por Carnelossi et al. (2004) de 15,2 ${ }^{\circ}$ Brix, porém equivalentes a $17,23{ }^{\circ}$ Brix, encontrado por Souza et al. (2007), trabalhando com frutos de diferentes clones de mangabeira. De acordo com Chitarra \& Chitarra (2005) o teor de açúcares normalmente constitui cerca de $85 \%$ do teor de sólidos solúveis; assim, os frutos com teores de sólidos solúveis mais elevados são preferidos tendo em vista o consumo in natura e o processamento, por acarretar maior rendimento, menor custo operacional e excelente grau de doçura (Pereira et al., 2000); merecem destaque, portanto, no que se refere a esta característica, as matrizes 3, 6 e 8 .

Tabela 4. Características físico-químicas da Hancornia speciosa (mangaba)

\begin{tabular}{ccccc}
\hline Matriz & pH & $\begin{array}{c}\text { SS } \\
\text { ('Brix) }\end{array}$ & $\begin{array}{c}\text { AT } \\
\text { (\% ác. cítrico) }\end{array}$ & $\begin{array}{c}\text { SS/AT } \\
\text { (Razão) }\end{array}$ \\
1 & $4,10 \mathrm{~b}$ & $15,80 \mathrm{c}$ & $0,86 \mathrm{c}$ & $18,54 \mathrm{bc}$ \\
2 & $4,05 \mathrm{bc}$ & $17,91 \mathrm{ab}$ & $0,88 \mathrm{c}$ & $20,39 \mathrm{~b}$ \\
3 & $4,19 \mathrm{~b}$ & $18,00 \mathrm{ab}$ & $0,64 \mathrm{~d}$ & $28,80 \mathrm{a}$ \\
4 & $4,39 \mathrm{a}$ & $15,34 \mathrm{c}$ & $0,61 \mathrm{~d}$ & $25,59 \mathrm{a}$ \\
5 & $3,91 \mathrm{c}$ & $15,92 \mathrm{c}$ & $1,00 \mathrm{bc}$ & $15,96 \mathrm{~cd}$ \\
6 & $4,03 \mathrm{bc}$ & $18,20 \mathrm{ab}$ & $1,18 \mathrm{a}$ & $16,02 \mathrm{~cd}$ \\
7 & $3,91 \mathrm{c}$ & $15,44 \mathrm{c}$ & $1,01 \mathrm{bc}$ & $15,54 \mathrm{~cd}$ \\
8 & $3,45 \mathrm{e}$ & $18,70 \mathrm{a}$ & $1,24 \mathrm{a}$ & $15,17 \mathrm{~cd}$ \\
9 & $3,62 \mathrm{~d}$ & $17,92 \mathrm{ab}$ & $1,22 \mathrm{a}$ & $14,76 \mathrm{~d}$ \\
10 & $3,65 \mathrm{~d}$ & $17,16 \mathrm{~b}$ & $1,11 \mathrm{ab}$ & $15,48 \mathrm{~cd}$ \\
MG & 3,93 & 17,04 & 0,98 & 18,62 \\
CV\% & 2,97 & 4,48 & 11,38 & 13,36 \\
\hline
\end{tabular}

Médias seguidas de letras diferentes nas colunas diferem pelo teste de Tukey, a nível de 0,05 de significância. SS - Sólidos solúveis; AT - Acidez titulável
A média da acidez titulável foi 0,98 , com variação de 0,61 a 1,24\% (Tabela 4); esses valores fazem com que o fruto de mangaba tenha sabor moderado e bem aceito para o consumo como fruta fresca haja vista que, de acordo com Sacramento et al. (2007) uma fruta que apresenta teores de ácido cítrico entre 0,08 e $1,95 \%$, pode ser classificada de sabor moderado e bem aceita para o consumo da fruta fresca. Souza et al. (2007) verificaram que os frutos da mangabeira apresentaram acidez média elevada de $1,77 \%$ oscilando entre 1,52 e $2,07 \%$ que, juntamente com um teor de SS, também alto confere, ao fruto, um sabor agridoce excelente proporcionando propriedades para sua conservação e industrialização; já Carnelossi et al. (2004) referenciaram um teor de acidez de ,070\% e Carvalho et al. (2003) mencionaram um valor de $1,48 \%$ de acidez em frutos da região do Conde (BA).

As matrizes apresentaram, na relação SS/AT, uma variação de 14,76 a 28,80 , ou seja, a média geral de 18,62, como mostra a Tabela 4. Souza et al. (2007) encontraram valores para SS/AT que variaram entre 5,78 e 12,69 sendo que a média geral foi 9,85 , valores inferiores aos encontrados no presente trabalho. A relação SS/AT que, de acordo com Chitarra \& Chitarra (2005) relaciona a qualidade do fruto em termos de maturidade e sabor, mostrou que a mangaba apresentou valor médio muito elevado indicando que a polpa desta fruta é indicada para a industrialização de produtos adocicados, tais como, doces, geleias, picolés e sorvetes; entretanto, o valor foi devido à baixa acidez e ao elevado teor de sólidos solúveis (Nascimento et al., 2011).

A variação nos dados de frutos ocorrida entre as matrizes avaliadas foi uma variação prevista, segundo Nascimento et al. (2011) por se tratar de plantas ainda não domesticadas.

Para as características físico-químicas dos frutos de mangabeira pôde-se observar que as correlações $\mathrm{pH} \mathrm{x}{ }^{\circ} \mathrm{Brix}(-$ $0,476) \mathrm{e}^{\circ}$ Brix x Acidez $(0,460)$ não foram significativas (Tabela 5) porém foi significativa a correlação do $\mathrm{pH}$ x Acidez (-0,872), apresentando correlação negativa, sinal de que o aumento do $\mathrm{pH}$ é inversamente proporcional ao aumento da acidez.

Tabela 5. Correlação para as características físicoquímicas dos frutos das matrizes de mangabeira

\begin{tabular}{lcc}
\hline \multicolumn{1}{c}{ Correlação } & Coeficiente de correlação (r) & Significância \\
pH $\times{ }^{\circ}$ Brix & $-0,476$ & ns \\
pH $\times$ Acidez & $-0,872$ & ${ }^{\star \star}$ \\
${ }^{\circ}$ Brix $\times$ Acidez & 0,460 & ns \\
\hline
\end{tabular}

** Significativo a nível de 0,01 de probabilidade $(p<0,01)$, ${ }^{\text {ns }}$ - Não-significativo

\section{Conclusões}

1. As avaliações físicas e físico-químicas sugerem que as matrizes estudadas apresentam variações estatisticamente significativas referentes a todas as variáveis avaliadas. Portanto, as matrizes apresentam alta variabilidade demonstrando que a população tem alto potencial genético para estudos de melhoramento.

2. Pode-se destacar os aspectos físico-químicos das matrizes como atraentes ao consumo como fruto fresco, tal como para industrialização. 


\section{Agradecimentos}

À Universidade do Estado da Bahia - UNEB e à Fundação de Amparo à Pesquisa do Estado da Bahia - FAPESB.

\section{Literatura Citada}

AIBA - Associação dos Irrigantes do Oeste da Bahia. Região Oeste da Bahia - Safra 2004. Informativo.

AOAC - Association of Official Agricultural Chemists. Official methods of analysis of the association of agricultural chemists, 11.ed., Washington: AOAC, 1990. 1141p.

Ávila, R.; Oliveira, L. F.; Ascheri, D. P. R. Caracterização dos frutos nativos dos cerrados: araticum, baru e jatobá. Revista Agrotecnologia, v.1, p.53-69, 2010.

Azeredo, H. M. C.; Brito, E. S. Tendências em conservação de alimentos. In: Azeredo, H. M. C. Fundamentos de estabilidade de alimentos. Fortaleza: Embrapa Agroindústria Tropical, 2004. p.135-150.

Borges, M. F.; Filgueiras, H. A. C.; Moura, C. F. H. Mangaba (Hancornia speciosa Gomes). In: Alves, R. E.; Filgueira, H. A. C.; Moura, C. F. H. (eds.). Caracterização de frutas nativas da América Latina. Jaboticabal: FUNEP, 2000. cap. 12, p.44-45. Série Frutas Tropicais.

Carnelossi, M. A. G.; Toledo, W. F. F.; Souza, D. C. L.; Lira, M. de L.; Silva, G. F. da; Jalali, V. R. R.; Viégas, P. R. de A. Conservação pós-colheita de mangaba (Hancornia speciosa Gomes). Ciência e Agrotecnologia, v.28, p.1119-1125, 2004.

Carvalho, J. E. U.; Nazaré, R. F. R.; Oliveira, W. M. Características físicas e físico-químicas de um tipo de bacuri (Platonia insignis Mart.) com rendimento industrial superior. Revista Brasileira de Fruticultura, v.25, p.326-328, 2003.

Chitarra, M. I. F.; Chitarra, A. B. Pós-colheita de frutos e hortaliças: Fisiologia e manuseio. Lavras: ESAL/FAEPE, 2005. 320p.

Cruz, E. D.; Carvalho, J.E. U. Biometria de frutos e germinação de sementes de Couratari stellata A. C. Smith (Lecythidaceae). Acta Amazônica, v.3, p.381-388, 2002.

Fagundes, G. R.; Yamanishi, O. K. Características físicas e químicas de frutos de mamoeiro do grupo solo comercializado em 4 estabelecimentos de Brasília DF. Revista Brasileira de Fruticultura, v.23, p.541-545, 2001.

Ganga, R. M. D.; Chaves, L. J.; Naves, R. V. Parâmetros genéticos em progênies de Hancornia speciosa Gomes do Cerrado. Scientia Forestalis, v.37, p.395-404, 2009.

Ganga, R. M. D.; Ferreira, G. A.; Chaves, L. J.; Naves, R. V.; Nascimento, J. L. do. Caracterização de frutos e árvores de populações naturais de Hancornia speciosa Gomes do Cerrado. Revista Brasileira de Fruticultura, v.32, p.101-113, 2010.

Hansen, O. A. de S.; Cardoso, R. L.; Fonseca, A. A. O.; Viana, E. de S.; Hansen, D. de S.; Barreto, N. S. E. Desenvolvimento e avaliação da estabilidade de néctar de mangaba. Magistra, v.25, n.2, p.148-156, 2013.
IAL - Instituto Adolfo Lutz. Métodos físico-químicos para análise de alimentos. 4.ed. Brasília: Ministério da Saúde, Agência Nacional de Vigilância Sanitária, 1985. 1141p.

Lederman, I. E.; Silva júnior, J. F.; Bezerra, J. E. F.; Espíndola, A. C. M. Mangaba (Hancornia speciosa Gomes). Jaboticabal: FUNEP, 2000. 35p.

Lima, E. D. P. de A.; Lima, C. A. de. A.; Aldrigue, M. L.; Gondim, P. J. S. Caracterização física e química dos frutos da umbucajazeira (Spondias spp.) em cinco estádios de maturação da polpa congelada e néctar. Revista Brasileira de Fruticultura, v.24, p.338-343, 2002.

Moura, C. F. H.; Alves, R. E.; Filgueiras, H. A. C.; Araújo, N. C. C.; Almeida, A. S. Quality of fruits native to latin america for processing: mangaba (Hancornia speciosa Gomes). Acta Horticulturae, v.2, p.549-554, 2002.

Nascimento, R. S. M.; Cardoso, J. A.; Oliveira, L. D. de; Oliveira, J. da S.; Cocozza, F. del M.; Cortes, J. M. Caracterização físico-química de muricis (Byrsonima verbascifolia Rich. ex A. Juss.) produzidos na região Oeste da Bahia. Magistra, v.23, p.236-242, 2011.

Pereira, M. C. T.; Salomão, L. C. C.; Mota, W. F. da; Vieira, G. Atributos físicos e químicos de frutos de oito clones de jabuticabeiras. Revista Brasileira de Fruticultura, v.22, p.16-21, 2000.

Sacramento, C. K. do; Matos, C. B.; Souza, C. N.; Barretto, W. S.; Faria, J. C. Características físicas, físico-químicas e químicas de cajás oriundos de diversos municípios da região sul da Bahia. Magistra, v.19, p.283-289, 2007.

Santos, F. S.; Paula, R. C.; Sabonaro, D. Z.; Valadares, J. Biometria e qualidade fisiológica de sementes de diferentes matrizes de Tabebuia chrysotricha (Mart. ex A. DC.) Standl. Scientia Forestalis, v.37, p.163-173, 2009.

Santos, J. T. S.; Soares, F. S. C.; Soares, D. S. C.; Campos, A. F. P.; Carnelossi, M. A. G.; Nunes, T. P.; Júnior, A. M. O. Avaliação de mangaba liofilizada através de parâmetros físico-químicos. Scientia Plena, v.8, p.1-5, 2012.

Souza, F. G. de; Figueredo, R. W. de; Alves, R. E.; Maia, G. A.; Araújo, I. A. de. Qualidade pós-colheita de frutos de diferentes clones de mangabeira (Hancornia speciosa Gomes). Ciência e Agrotecnologia, v.31, p.1449-1454, 2007.

Venturini Filho, W. G. Bebidas alcoólicas - Ciência e tecnologia. São Paulo: Blucher. 2010. 461p.

Vieira, F. A.; Gusmão, E. Biometria, armazenamento de sementes e emergência de plântulas de Talisia esculenta Radlk (Sapindaceae). Ciência e Agrotécnica, v.32, p.10731079, 2008.

Vieira Neto, R. D. Recomendações técnicas para o cultivo da mangabeira, Aracaju: Embrapa Tabuleiros Costeiros, 2001. 26p. Circular Técnica, 20. 